\title{
Insulation of a synthetic hydrogen metabolism circuit in bacteria
}

\author{
Christina M Agapakis', Daniel C Ducat ${ }^{1,2}$, Patrick M Boyle ${ }^{1}$, Edwin H Wintermute', Jeffrey C Way ${ }^{2}$, \\ Pamela A Silver ${ }^{1,2^{*}}$
}

\begin{abstract}
Background: The engineering of metabolism holds tremendous promise for the production of desirable metabolites, particularly alternative fuels and other highly reduced molecules. Engineering approaches must redirect the transfer of chemical reducing equivalents, preventing these electrons from being lost to general cellular metabolism. This is especially the case for high energy electrons stored in iron-sulfur clusters within proteins, which are readily transferred when two such clusters are brought in close proximity. Iron sulfur proteins therefore require mechanisms to ensure interaction between proper partners, analogous to many signal transduction proteins. While there has been progress in the isolation of engineered metabolic pathways in recent years, the design of insulated electron metabolism circuits in vivo has not been pursued.

Results: Here we show that a synthetic hydrogen-producing electron transfer circuit in Escherichia coli can be insulated from existing cellular metabolism via multiple approaches, in many cases improving the function of the pathway. Our circuit is composed of heterologously expressed [Fe-Fe]-hydrogenase, ferredoxin, and pyruvateferredoxin oxidoreductase (PFOR), allowing the production of hydrogen gas to be coupled to the breakdown of glucose. We show that this synthetic pathway can be insulated through the deletion of competing reactions, rational engineering of protein interaction surfaces, direct protein fusion of interacting partners, and co-localization of pathway components on heterologous protein scaffolds.
\end{abstract}

Conclusions: Through the construction and characterization of a synthetic metabolic circuit in vivo, we demonstrate a novel system that allows for predictable engineering of an insulated electron transfer pathway. The development of this system demonstrates working principles for the optimization of engineered pathways for alternative energy production, as well as for understanding how electron transfer between proteins is controlled.

\section{Background}

Metabolic electron transfer is a well-characterized process [1], understood at the level of engineering principles [2]. Electrons readily tunnel between iron-sulfur clusters, whose electrical potential is defined by the chemical environment created by the amino acids that surround them in the protein matrix [3]. As with other biological systems, including signal transduction [4], complex electron transfer pathways likely evolved through gene duplication, recombination, and drift of iron-sulfur containing proteins, with interaction between two iron sulfur containing proteins or domains largely controlled by electrostatic forces [5]. Taking advantage

\footnotetext{
* Correspondence: pamela_silver@hms.harvard.edu
'Department of Systems Biology, Harvard Medical School, Boston, MA 02115,

* Correspondence: pamela_silver@hms.harvard.edu
'Department of Systems Biology, Harvard Medical School, Boston, MA 02115, USA
}

(ㄷ) 2010 Agapakis et al; licensee BioMed Central Ltd. This is an Open Access article distributed under the terms of the Creative Commons Attribution License (http://creativecommons.org/licenses/by/2.0), which permits unrestricted use, distribution, and reproduction in any medium, provided the original work is properly cited. of the modular nature of iron sulfur protein interactions, several groups have recombined redox proteins in vitro in order to engineer novel electron transfer pathways [6]. Much of the focus in previous work has been to create physical interfaces between electrodes and enzymes [7], joining electron transfer proteins with electron-generating proteins through "molecular Lego" in vitro [8].

There are many appealing applications for such engineered electron transfer systems in vitro, such as miniaturized biofuel cells, biocatalysts, and biosensors [7]. These approaches, however, do not take advantage of the self-assembly and self-regenerating abilities of live cells. Engineered cellular pathways in vivo have the potential to impact our understanding of cellular electron transfer systems in live cells and may provide self-renewing 
platforms for the continuous production of fuels and other useful molecules [9].

To this end, we chose to design a synthetic electron transfer circuit in E. coli that utilizes [FeFe]-hydrogenases, a class of metalloenzymes that catalyse the reversible reduction of protons to molecular hydrogen [10]. Previous research using hydrogenases within engineered electron transfer pathways have relied heavily on in vitro approaches. For example, hydrogenase enzymes have been explored as tools for hydrogen fuel production by purified enzyme cocktails in vitro [11]. Alternatively, the hydrogenase active site has been modeled synthetically for use in fuel cells as a catalytic center that does not require rare metals to function [12]. While these in vitro systems are inherently insulated from natural metabolism, they suffer from the same drawbacks as other in vitro enzymatic processes in the difficulty of production and purification, and lack of robustness from the living cell. Metabolic [13] and protein engineering [14] of natural hydrogen production pathways in $E$. coli have yielded improvements in hydrogen yield, but these methods are limited in the substrate specificity of the native [NiFe]-hydrogenases [15]. Synthetic pathways expressing hydrogenases along with exogenous electron donors and carriers can be used to supplement and optimize hydrogen production from E. coli, as well as improve our understanding of electron transfer pathways.

Natural hydrogen metabolism pathways in a variety of prokaryotic and eukaryotic species can either consume hydrogen as a source of low potential electrons, or produce hydrogen as a sink for reducing equivalents generated during anaerobic fermentation. In E. coli, both hydrogen consumption [16] and production [17] are performed by several $[\mathrm{NiFe}]$-hydrogenases. While the native E. coli [NiFe]-hydrogenases are coupled to NADH, with a reducing potential of $-320 \mathrm{mV}$, [FeFe]-hydrogenases are partnered with the electron carrying protein ferredoxin, which can have a significantly stronger reducing potential, typically close to that of the $\mathrm{H}_{2} / \mathrm{H}^{+}$pair $(-420 \mathrm{mV})$ [10]. As a result, $[\mathrm{FeFe}]$-hydrogenases thermodynamically favor hydrogen production. Moreover, their high hydrogen production activity, conserved structure, and relatively simple maturation pathway make [FeFe]-hydrogenases excellent enzymatic modules for recombinant expression in a synthetic system. Furthermore, heterologously expressed [FeFe]-hydrogenases from several species have been characterized in vitro [18] and in vivo [19]

Heterologous expression of [FeFe]-hydrogenase alone is sufficient for small, measurable hydrogen production from E. coli in vivo, and this hydrogen production is increased with the co-expression of ferredoxins from several organisms [19]. Furthermore, overexpression of a ferredoxin oxidoreductase can link hydrogen production to cellular metabolism [20]. Pyruvate-ferredoxin oxidoreductase (PFOR) from several anaerobic or microaerobic species of microorganisms reduces ferredoxin as it breaks down pyruvate to acetyl-CoA. Coexpression of PFOR, ferredoxin, and [FeFe]-hydrogenase therefore couples the breakdown of glucose with the establishment of a reduced ferredoxin pool (Figure 1A). As $[\mathrm{FeFe}]$-hydrogenases are efficient electron acceptors from ferredoxin, co-expressed [FeFe]-hydrogenases within this system can reset the redox state of the ferredoxin pool, thereby completing the circuit, and providing a readout of electron flux through the pathway as a function of hydrogen production.

Synthetic biology circuits such as the above-described PFOR-ferredoxin-hydrogenase pathway must be integrated into cellular metabolism in order to function, but must also be insulated from competing reactions in order to optimize output and ensure proper behavior. Natural electron transfer pathways are insulated in several ways that can be adapted for use in synthetic circuits (Figure 1B). Elimination of competing reactions, through gene deletion, transcriptional regulation, or spatial separation into different subcellular compartments, can isolate proteins from competition for electrons. For example, the expression of hydrogenase genes from many organisms are transcriptionally regulated by the presence of oxygen [21], likely in order to prevent competition for electrons with aerobic metabolism.

Isolation of electron transfer pathways can also evolve through physical changes to proteins, either through point mutations that alter interaction surfaces between two redox partners, or adaptations that co-localize interaction partners to one another or to secondary scaffolding complexes. The coevolution of protein interaction surfaces has been postulated to play a role in the control of bacterial signal transduction pathways [22] and is likely involved in the evolution of many iron-sulfur containing proteins capable of interacting with ferredoxins [23]. Isolation of ironsulfur proteins through physical scaffolding, either in the mitochondrial or chloroplast membranes or through direct protein fusion, is thought to play a large role in the evolution of complex electron transfer pathways as well a the iron-sulfur proteins themselves. For example, larger [FeFe]-hydrogenases, such as those from Clostridium species contain several "ferredoxin-like" domains [24]. It is speculated that these domains arose through ancestral gene fusions, enhancing hydrogenase interaction with other ferredoxins, and providing an electron transport channel towards the hydrogenase active site.

Herein, we create and characterize a synthetic electron transfer circuit that couples hydrogen evolution with the breakdown of glucose in $E$. coli via heterologous expression of PFOR, Ferredoxin, and [FeFe]-hydrogenase. We used the modular nature of our synthetic circuit to recapitulate several of the aforementioned isolation strategies 


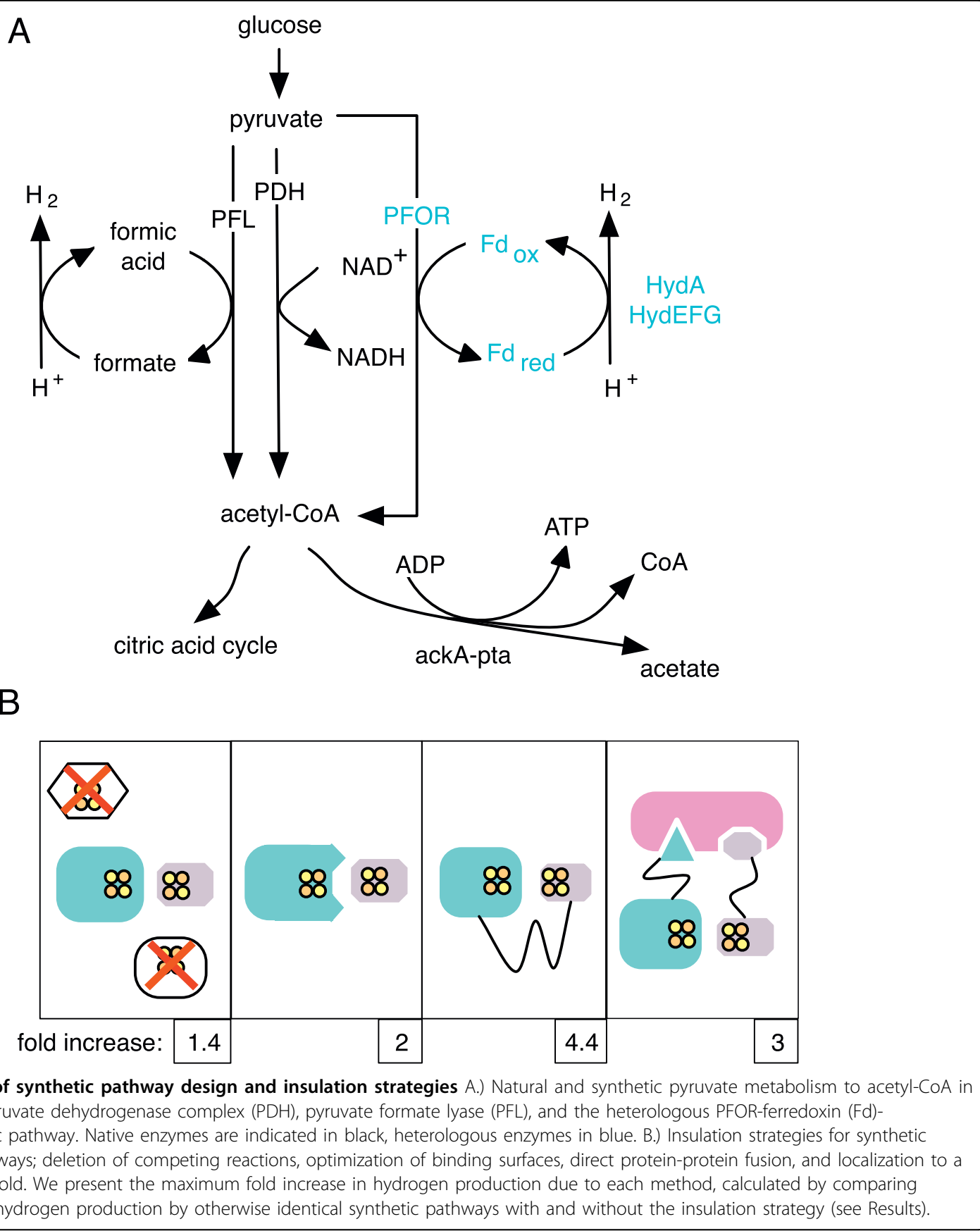

for electron transfer pathways. We reproduced spatiotemporal isolation through the deletion of competing iron-sulfur proteins. We explored the interaction surface of the hydrogenase and ferredoxin, testing four mutations of surface amino acids of the [FeFe]-hydrogenase from Chlamydomonas reinhardtii previously predicted to improve ferredoxin binding [25]. We synthetically modeled physical scaffolding of electron transfer proteins, both through direct protein fusion of the Clostridium acetobutylicum hydrogenase and ferredoxins with flexible peptide linkers, and through connection of hydrogenase and ferredoxin to a heterologous protein scaffold [26]. All of these insulation strategies significantly affected the function of our synthetic circuit, in many cases increasing total hydrogen production. The highest improvement was seen with direct protein-protein fusion of the hydrogenase and ferredoxin, with an optimal linker length increasing hydrogen production by up to four fold. This method is easily transferrable to other synthetic electron transfer pathways and may provide clues to 
understanding the evolution of complex electron transfer proteins with multiple ferredoxin-like domains.

\section{Materials and methods Plasmids and cloning}

All cloning was done in E. coli DH5 $\alpha$. Hydrogenase genes from Chlamydomonas reinhardtii and the ferredoxin I gene from Spinacia olearcea were commercially synthesized by Codon Devices (Cambridge, MA), codon optimized for expression in Saccharomyces cerevisiae and acceptable for use in $E$. coli for wide applicability (see Additional file 1, figure S1 for nucleotide sequences). Hydrogenases from Clostridium acetobutylicum and Clostridium saccharobutylicum were cloned from plasmids received from Matthew Posewitz (National Renewable Energy Laboratory, Golden, CO). Hydrogenase genes HydA and HydB were cloned from Shewanella oneidensis using colony PCR of bacterial cultures from Colleen Hansel (Harvard University, Cambridge, MA). Thermotoga maritima HydA was cloned from genomic DNA provided by Kenneth Noll (University of Connecticut, Storrs, CT). PFOR and ferredoxin [27] were cloned from Clostridium acetobutylicum genomic DNA (ATCC, Manassas, VA). Zea mays ferredoxin was cloned from genomic DNA isolated from corn using DNeasy Plant Mini Kit (Qiagen, Valencia, CA). PFOR from Desulfovibrio africanus was isolated from plasmid pLP1 [28] provided by Laetitia Pieulle (Centre National de la Recherche Scientifique, Marseille, France) and $y d b K$ was obtained through colony PCR of E. coli BL21. Plasmids for tetracycline-responsive expression of synthetic scaffold proteins were provided by John Dueber [26] (University of California, Berkeley), and ferredoxin/hydrogenase fusions to metazoan GBD, SH3, and PDZ ligand domains were constructed, bridged by flexible $\left(\mathrm{Gly}_{4} \mathrm{Ser}\right)_{\mathrm{x}}$ linkers. Oligonucleotides for metazoan ligand domains and linker sequences were purchased from Integrated DNA Technologies, with ligand sequences identical to those previously reported [26].

Site directed mutagenesis of the Clostridium and Shewanella hydrogenase genes to remove restriction sites needed for cloning and for alteration of C. reinhardtii HydA ferredoxin binding surface was carried out using the Quikchange Multi-Site Directed Mutagenesis Kit according to manufacturer instructions (Stratagene, La Jolla, CA). Cloning and mutagenesis primers are listed in Additional file 1, Table S1 (Integrated DNA Technologies, Coralville, IA)

Cloning of hydrogenase-ferredoxin fusion proteins was done using BioBrick standard assembly [29] and subsequently cloned into Novagen Duet vectors (Novagen, Gibbstown, NJ) whose multiple cloning sites were mutated to accept BioBrick parts (Additional file 1, figure S2). Strep-II tag (WSHPQFEK), (Gly $\left.{ }_{4} \mathrm{Ser}\right)_{2}$, and (Gly $\mathrm{Ger}_{4}{ }_{4}$ oligonucleotides with flanking BioBrick sites were purchased from Integrated DNA Technologies. Longer $\left(\mathrm{Gly}_{4} \mathrm{Ser}\right)$ linkers were made through BioBrick fusion of multiple linker sequences or through PCR amplification from other chimeric proteins [30].

\section{Protein expression}

All protein expression and hydrogenase activity assays were performed in E. coli BL21 (DE3). Cells were transformed with modified pCDF-duet with $C$. reinhardtii HydEF in MCS1 and C. reinhardtii HydG in MCS2, and with modified pACYC-duet with C. acetobutylicum PFOR or E. coli ydbK in MCS1 or Desulfovibrio vafricanus PFOR cloned into the downstream NdeI and AvrII sites of MCS2. Hydrogenase/ferredoxin pairs were transformed either in each multiple cloning site of modified pET-duet, or for the S. oneidensis hydrogenase HydA in MCS1, HydB in MCS2, and ferredoxin in MCS1 of modified pCOLA-duet. To compare in vitro hydrogen production using maturation factors from Clostridium acetobutilicum, we used plasmids provided by Matthew Posewitz (pCDF-duet with CaHydE in MCS1, CaHydF in MCS2 and pET-duet with CaHydA in MCS1 and CaHydG in MCS2 [18]). Artificial scaffolds were expressed from pJD plasmids provided by John Dueber, previously described in Dueber et. al. 2009 [26].

\section{E. coli Gene Deletion}

Hydrogenase knockout ( $\triangle h y c E, \triangle h y a B, \Delta h y b C)$ and $\Delta f p r, \Delta y d b K, \Delta h c r, \Delta y e a X, \Delta h c a D$, or $\Delta f r d B$ single deletion strains were made by sequential P1 transduction from the Keio collection [31] into BL21(DE3) $\Delta$ tonA, followed by removal of the $\operatorname{Kan}^{\mathrm{R}}$ marker by standard procedures.

\section{SDS-Page and Western Blotting}

E. coli cells were lysed with Bacterial Protein Extraction Reagent (B-PER, Pierce, Rockford, IL), protein samples were normalized using the Bradford assay (Bio-Rad, Hercules, CA), diluted into SDS-PAGE loading buffer and loaded onto a 4-20\% Tris/glycine/SDS acrylamide gel. $\alpha$-Strep-tag II antibody (HRP-conjugated, Novagen, Gibbstown, NJ) or $\alpha$-ferredoxin primary antibody (Agrisera, Vännäs, Sweden) and $\alpha$-Rabbit IgG secondary antibody were used.

\section{Hydrogen production assays}

Bacterial cultures were grown aerobically for two hours until reaching an $\mathrm{OD}_{600}$ of approximately 0.15 in $\mathrm{LB}$ media with appropriate antibiotic $(50 \mu \mathrm{g} / \mathrm{ml}$ ampicillin, $25 \mu \mathrm{g} / \mathrm{ml}$ spectinomycin, $25 \mu \mathrm{g} / \mathrm{ml}$ kanamycin, and/or $12.5 \mu \mathrm{g} / \mathrm{ml}$ chloramphenicol) in $40 \mathrm{ml}$ glass serum vials, induced with $1 \mathrm{mM}$ IPTG (and $2 \mu \mathrm{g} / \mathrm{ml}$ anhydrous tetracycline when relevant for the induction of scaffold proteins) and sparged with argon. For the methyl 
viologen assay, adapted from King et. al. [18], vials were sparged for 2 hours and then lysed with assay buffer containing $50 \mathrm{mM}$ Tris pH 7.0, 50\% B-PER, $10 \mathrm{mM}$ methyl viologen (Sigma, St. Louis, MO) and $50 \mathrm{mM}$ sodium dithionite (Fisher, Pittsburgh, PA), the vials were capped with rubber septa, the cells were vortexed and allowed to rock overnight at room temperature. Hydrogen concentration in the headspace gas was measured by gas chromatography (Shimadzu GC-14A). In vivo hydrogen production assays were performed in a similar fashion, except that cultures were supplemented with $0.5 \%$ glucose at the time of IPTG induction, sparged for 30 minutes and simply capped and shaken ovenight at $37^{\circ} \mathrm{C}$ before measuring headspace gas composition. Glucose curves were measured in cells pretreated overnight with $1 \mathrm{mM}$ IPTG then immediately diluted into LB + variable glucose $+1 \mathrm{mM}$ IPTG, then sparged and grown overnight. All hydrogen production values were normalized to an $\mathrm{OD}_{600}$ of 0.15 .

\section{Homology modeling}

Homology model of $C$. reinhardtii HydA1 was made using the SWISS-MODEL [32] server with the Clostridium pasteurinium HydA X-ray structure (1FEH [24]) as a template.

\section{Results}

\section{In vitro hydrogen production from heterologously} expressed hydrogenases

To create a synthetic electron metabolism circuit with hydrogenase as the terminal electron acceptor, we first investigated the activity of various hydrogenase genes heterologously expressed in the presence of appropriate maturation factors. We adapted a previously established in vitro hydrogenase activity assay [18], and measured hydrogen production from crude lysates of bacteria expressing hydrogenases and maturation factors from several species in the presence of a chemical electron donor, methyl viologen. Previous reports have shown that the hydrogenase maturation factors from C. reinhardtii, HydEF and HydG, are unstable when heterologously expressed in E. coli [18], likely due to the genes' high GC content, while the maturation factors from Clostridium acetobutylicum were able to mature [FeFe]-hydrogenases from a wide range of species. Using commercially synthesized, codon optimized maturation factors from $C$. reinhardtii we were able to alleviate the instability of the gene constructs. We found that in vitro hydrogen production from the Clostridium acetobutylicum hydrogenase was identical when coexpressed with the synthetic maturation factors or with HydE, HydF, and HydG from C. acetobutylicum (data not shown). All subsequent experiments were performed using the optimized C. reinhardtii maturation factors.
We compared the in vitro hydrogen production of [Fe-Fe] hydrogenases from Clostridium acetobutylicum, Clostridium saccharobutylicum, Chlamydomonas reinhardtii, Shewanella oneidensis, and Thermotoga maritima, all of which are homologous in their catalytic domain (Additional file 1, figure S3). All hydrogenases except HydA from Thermotoga maritima could be expressed at a high level in E. coli (figure 2A), and were functional in vitro (figure 2B). Hydrogen levels increased linearly for the first several hours of measurement (data not shown), and we found that levels of hydrogen gas in the headspace after overnight incubation correlated to the relative rate of hydrogenase activity during this linear phase. Our overnight in vitro results agree with previous reports of in vitro hydrogen production rates, with the hydrogenases from Clostridium species producing the highest levels of hydrogen [18]. The heterologously expressed hydrogenase from Shewanella oneidensis is functional at relatively low levels in vitro when both subunits are coexpressed in $E$. coli with maturation factors from C. reinhardtii.

The in vitro assay is useful to test and compare the activities of heterologously expressed hydrogenase genes, but as the assay uses an exogenous reducing agent, it does not provide information on the electron flux within normal metabolic pathways in vivo. To measure electron flux in vivo as a function of hydrogen production, hydrogenase activity must be integrated into a functional electron transfer pathway. One well established class of electron donors to hydrogenases are ferredoxins, small soluble proteins that contain iron-sulfur clusters. Construction of a system where hydrogenase activity depends on electron transfer from ferredoxin would allow for comparison to in vitro data to provide information on hydrogenase behavior and hydrogenase-ferredoxin interaction dynamics.

\section{In vivo construction and optimization of a synthetic hydrogen-producing circuit}

To produce hydrogen in vivo from glucose, the [FeFe]hydrogenase was coexpressed with its required maturation factors, ferredoxin, and pyruvate-ferredoxin oxidoreductase (PFOR) from different species. In this heterologous circuit, PFOR oxidizes pyruvate to acetyl-CoA, reducing ferredoxin, which then transfers the electron to the hydrogenase. In normal E. coli metabolism, the oxidative breakdown of pyruvate to acetyl-CoA is performed either aerobically by the pyruvate dehydrogenase complex, reducing $\mathrm{NAD}^{+}$, or anaerobically by pyruvate formate lyase, generating formate (figure 1A). PFOR functions in certain anaerobic bacteria and in eukaryotic parasites that possess hydrogenosomes, organelles evolutionarily related to the mitochondrion that generate a proton gradient through 

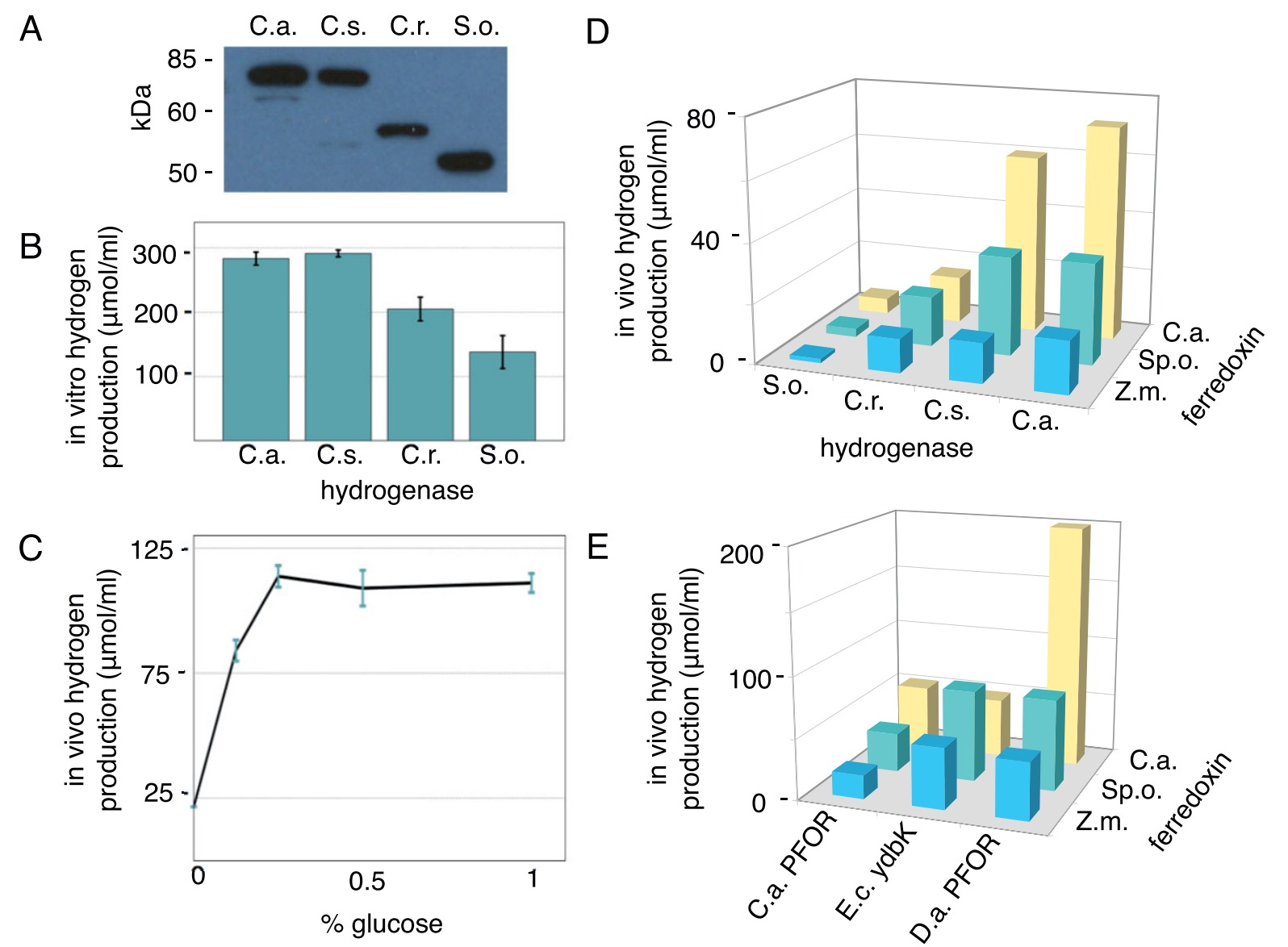

Figure 2 Characterization of synthetic hydrogen production pathway A.) Western blot of Strep-II tagged hydrogenase expression. B.) In vitro hydrogen production from E. coli strains expressing various hydrogenases, measured by the methyl viologen in vitro assay [18]. C.a. = C. acetobutylicum, C.s. = C. saccharobutylicum, C.r. = C. reinhardtii, S.o. = Shewanella oneidensis. C.) Glucose-dependence of hydrogen production. Here and below, in vivo and in vitro hydrogen production values are in units of $\mu \mathrm{mol}$ hydrogen $/ \mathrm{ml}$ of $E$. coli culture, normalized to an $\mathrm{OD}_{600}$ of 0.15 unless otherwise stated. Assays were performed in triplicate, with error bars indicating standard deviation. D.) In vivo hydrogen production from E. coli strains expressing all combinations of the four hydrogenases vs. three ferredoxins from C. acetobutylicum, Spinacia olearcea (Sp.o), and Zea mays (Zm). E.) In vivo hydrogen production from the C. acetobutylicum hydrogenase paired with combinations of three ferredoxins and three PFOR genes.

the production of hydrogen gas [33]. PFOR is an attractive electron source for a synthetic hydrogen production circuit as overexpression of a putative E. coli PFOR homolog, YdbK, increases in vivo hydrogen production by heterologously expressed [FeFe]-hydrogenase and ferredoxin [20], PFOR purified from Clostridium pasteurianum has been shown to reduce a number of ferredoxins in vitro [34], and functional PFOR from Desulfovibrio africanus has been recombinantly expressed in E. coli [28].

Consistent with the establishment of a synthetic electron transport circuit in vivo, we observed high levels of glucose-dependent hydrogen production upon coexpression of PFOR, hydrogenase and its maturation factors, and ferredoxin all from Clostridium acetobutylicum in an $E$. coli strain lacking endogenous hydrogenases ( $\Delta h y c E, \Delta h y a B, \Delta h y b C$, figure $2 C$ ). Hydrogen production was again measured after overnight incubation, as we found that hydrogen production in vivo from glucose was exhausted after 16 hours (data not shown). We were unable to detect hydrogen production in the parental strain of $E$. coli with the native hydrogenases deleted. Removal of any individual pathway component from the synthetic circuit drastically reduced in vivo hydrogen production. However, as has been previously reported, there was a small background level of hydrogen production from expression of hydrogenase and maturation factors alone [35]. Consistent with previous results [19], we found this background hydrogen production was slightly increased upon overexpression of ferredoxin in addition to hydrogenase, indicating that there are E. coli proteins capable of reducing both hydrogenases and plant-type ferredoxins, several 
candidate proteins of which we deleted in the following section (figure 3).

The hydrogenase-ferredoxin-PFOR pathway constitutes a modular system, where each element can be exchanged with homologous genes from different organisms. By coexpressing pathway enzymes from diverse microorganisms, we were able to compare the relative interaction strengths of four hydrogenases, three ferredoxins (figure 2D), and three PFORs (figure 2E). All ferredoxins were able to transfer electrons between PFOR and hydrogenases from different species with varying levels of efficiency.

In vivo hydrogen production from circuits expressing each of the four hydrogenases (C. acetobutylicum, C. saccharobutylicum, C. reinhardtii, and S. oneidensis) followed the same trend as the in vitro experiments, with the highest hydrogen production observed with the clostridial hydrogenases (figure 2D). The relative interaction and electron transfer rates for hydrogenase and ferredoxin were explored by comparing the in vivo hydrogen production of circuits made up from all pairwise combinations of the four hydrogenases and ferredoxin from C. acetobutylicum, Spinacea olearcea, and Zea mays and the PFOR from C. acetobutylicum (figure 2D). All hydrogenases produced the highest output when co-expressed with bacterial type 2-[4Fe-4S] ferredoxin from Clostridium acetobutylicum, with a potential of $-420 \mathrm{mV}$ [27]. Intermediate levels of hydrogen were produced using leaf-type [2Fe-2S]-ferredoxin I from spinach, S. olearcea $(-420 \mathrm{mV}$ [36]) while the homologous root-type ferredoxin III from corn, $Z$. mays (-345 $\mathrm{mV}$ [36]) led to significantly lower in vivo hydrogen levels in all cases. Interestingly, the difference in hydrogen production from circuits expressing bacterial versus plant-type ferredoxins was more significant for hydrogenases from bacterial species. Hydrogenase from C. reinhardtii, which naturally

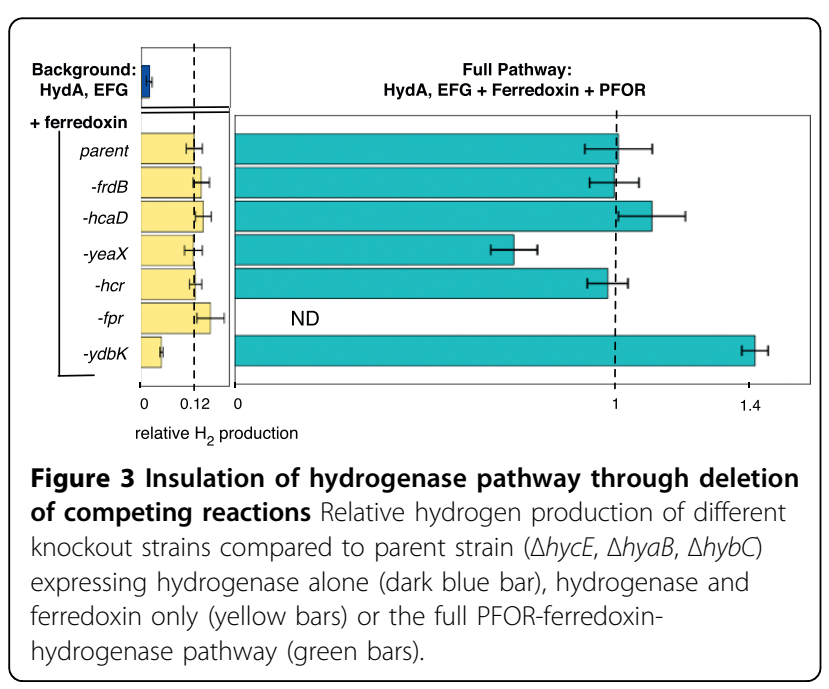

pairs with plant-type ferredoxins, produced similar levels of hydrogen when co-expressed with ferredoxin from C. acetobutylicum or S. olearcea (figure 2D).

The interaction of overexpressed PFOR from C. acetobutylicum, D. africanus, or the PFOR homolog YdbK from $E$. coli with the three ferredoxins was compared in a similar fashion in circuits containing the C. acetobutylicum hydrogenase (figure 2E). Overexpression of PFOR from $C$. acetobutylicum and YdbK from E. coli led to similar levels of hydrogen production, although surprisingly, the highest levels of hydrogen produced from YdbK occurred when it was coexpressed with plant-type ferredoxin from S. olearcea. Overall, the highest levels of hydrogen production were seen with the PFOR from $D$. africanus, coexpressed with the hydrogenase and ferredoxin from $C$. acetobutylicum.

\section{Isolation of the hydrogen producing circuit through deletion of competing reactions}

Natural biological electron transfer circuits are insulated to prevent electron leaks that can cause damage by creating oxygen radicals and insulated from one another to prevent "short circuiting" [37]. We sought to insulate our hydrogen producing circuit from competing metabolism to improve levels of hydrogen production and to better understand natural biological pathway isolation, a priority for the design of synthetic metabolic pathways. Although our constructed pathway is made up of genes that are divergent from $E$. coli metabolic enzymes, given the non-specific electrostatic interactions that mediate many ferredoxin interactions [5], native iron-sulfur proteins may interact with the proteins of the heterologous pathway. This is evidenced by the background hydrogen production in strains expressing only heterologous hydrogenases and ferredoxins (figure 3). Deletion of these potentially competing redox interaction partners should improve pathway function. To address these issues, we deleted six genes identified through their homology to plant-type ferredoxins or ferredoxin oxidoreductases that still allowed for viability $(f p r$, flavodoxin: $\mathrm{NADP}^{+}$reductase [19]; $y d b K$, the putative PFOR homo$\log [19] ; h c r$, an NADH oxidoreductase; yeaX, a predicted oxidoreductase; $h c a D$, ferredoxin: $\mathrm{NAD}^{+}$reductase; and $\mathrm{frdB}$, fumarate reductase. Additional file 1, figure S4).

These six deletions were tested individually in a $\triangle h y c E$, $\triangle h y a B, \triangle h y b C$ background while expressing hydrogenase from $C$. acetobutylicum and maturation factors from C. reinhardtii, ferredoxin from S. olearcea, with or without co-expression of PFOR from D. africanus. Deletion of $f p r$ and $y d b K$ have been previously shown to slightly decrease the background level of hydrogenase activity in vivo [19]. We found that only the $y d b K$ deletion had any significant effect on hydrogen production compared to the hydrogenase knockouts alone. The background level 
of hydrogen production from HydA and ferredoxin expressed alone was decreased by half in the $y d b K$ deletion strain, whereas hydrogen production from the full pathway with the $D$. africanus PFOR was increased by 1.4 fold (figure 3 ). This is consistent with our finding that overexpression of $y d b K$ led to high levels of electron transfer when co-expressed with ferredoxin from spinach, indicating that endogenous $y d b K$ is able to disrupt the synthetic electron transfer pathway.

\section{Insulation through mutation of the hydrogenase- ferredoxin interaction surface}

As an independent strategy, we attempted to insulate the pathway from competing electron metabolism through modification of the interaction surface of the hydrogenase and ferredoxin by rational protein design. Co-evolution of interacting protein pairs for preferential binding between natural partners likely plays a large role in the isolation of natural pathways [38], and this principle has been used in designing synthetic signal transduction systems [22]. To test whether mutations in a component of the artificial pathway specifically enhanced activity by improving the ferredoxin-hydrogenase interaction, we compared in vivo activity, which is ferredoxin-dependent, with in vitro activity, in which a chemical reducing agent, methyl viologen, drives hydrogen production. This in vitro assay thus measures ferredoxin-independent hydrogen production, reflecting the activity and expression level of the hydrogenase itself.

The interaction between ferredoxins and clostridial hydrogenases is poorly characterized, with evidence that more than simple electrostatic reactions may play an role in mediating the transfer of electrons [34]. In contrast, the interaction surface between the hydrogenase from Chlamydomonas reinhardtii and its cognate ferredoxin has been extensively modeled in silico, with evidence that this interaction has a strong electrostatic component $[39,40]$. Long et. al. [25] proposed a structural model for the interaction between the hydrogenase HydA2 from Chlamydomonas reinhardtii and its cognate $[2 \mathrm{Fe}-2 \mathrm{~S}]$ ferredoxin, suggesting several mutations that might enhance this interaction due to improved charge complementarity (figure 4A). Ferredoxin is rich in negatively charged residues, and the mutations, E5K, P2K, M119K, or D126K are designed to increase the positive charge of the hydrogenase binding surface. We tested these mutations using HydA1 from C. reinhardtii, and ferredoxin from spinach, both of which are closely related to the proteins studied in the in silico model (Additional file 1, figure S5). We found that two mutations in HydA1, D126K and E5K, improved in vivo hydrogen production while in vitro these mutations showed less or no effect (figure 4B). As in vitro hydrogen production was closely correlated to hydrogenase

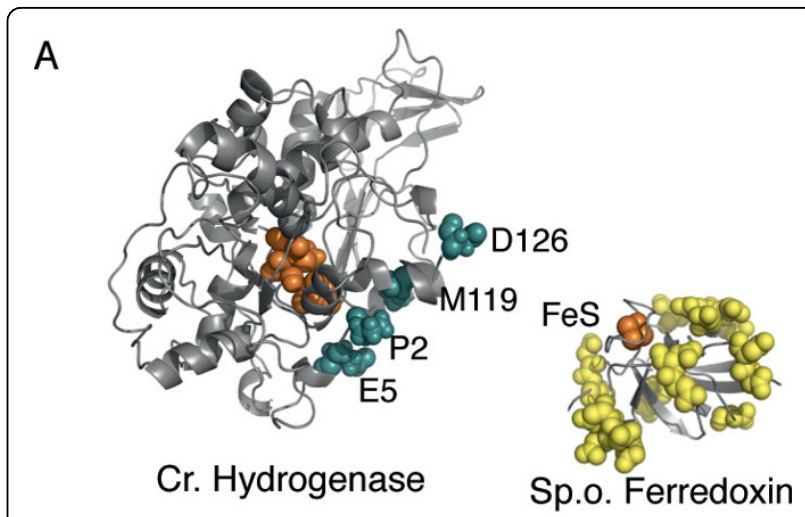

B

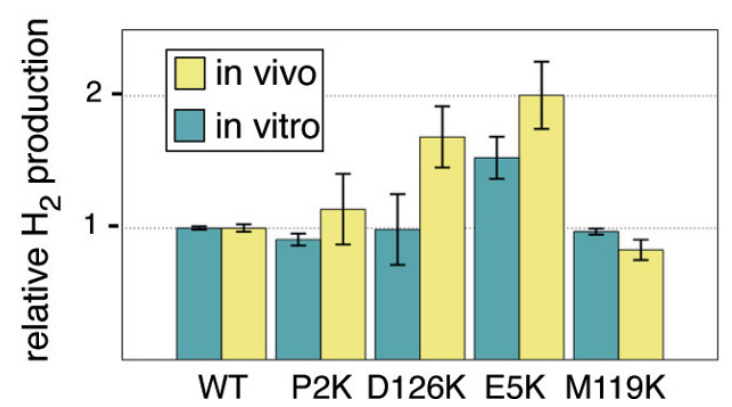

Figure 4 Insulation of the hydrogenase pathway through ferredoxin binding surface mutagenesis $\mathrm{A}$.) Homology model of C. reinhardtii hydrogenase with mutated residues highlighted in cyan and spinach ferredoxin X-ray structure (1A70 [54]) with negatively charged residues highlighted in yellow. Iron-sulfur clusters and the hydrogenase catalytic cluster are highlighted in orange. B.) Relative in vitro and in vivo hydrogen production for wild type and mutated C. reinhardtii hydrogenase. Mutants D126K and E5K, which enhance charge-complementarity at the putative interaction surface, show a specific enhancement of in vivo activity relative to activity changes seen in the ferredoxin-independent in vitro assay.

expression level (data not shown), the improvement in in vitro hydrogen production that was seen for E5K may be the result of increased protein expression.

\section{Improvement of hydrogen production activity through direct protein fusion}

Many metabolic electron transfer pathways are insulated through the physical scaffolding of protein components in the membrane, for example in the electron transport chain of mitochondria or chloroplasts. Additionally, some electron transport proteins themselves are built from combinations of modular electron binding domains, including the hydrogenase from Clostridium [24]. We sought to isolate our synthetic pathway and improve hydrogen production through physically linking the hydrogenase and ferredoxin in order to increase the chance of binding and electron transfer between the desired partners. We were able to show improved function of the artificial pathway through genetic fusion of 
the hydrogenase and ferredoxin. Chimeras between ferredoxin and various ferredoxin reductases have been shown to be functional in vitro [41], with improved electron transfer rates presumably due to the increased local concentration of reduced ferredoxin. We fused the hydrogenase from C. acetobutylicum (figure 5A) with ferredoxin from spinach (figure $5 \mathrm{~B} \& \mathrm{C}$ ) or from $C$. acetobutylicum (figure 5D) using flexible protein linkers of various lengths.

Our hydrogenase-spinach ferredoxin chimeric proteins were all expressed at a high level in E. coli (figure 5B) and active at identical levels in vitro (figure $5 \mathrm{C}$ ), regardless of the orientation of the fusion or linker length, consistent with the lack of a requirement for ferredoxin in the in vitro assay. In vitro data for fusions with the $C$. acetobutylicum ferredoxin followed a similar trend (data not shown). However, in vivo activity of the fusion proteins when coexpressed with the PFOR from $D$. africanus depended on linker length as well as overall configuration. Fusions of the spinach ferredoxin to the hydrogenase $\mathrm{C}$-terminus displayed decreased function when the linker is very short, a nearly five-fold improvement at intermediate length linkers, and activity falling gradually to the level of that of separate proteins at longer lengths. This length dependent behavior is consistent with models of changes in local concentration of reactants due to enzyme fusion [42]. Protein fusion to the $\mathrm{C}$-terminus of the spinach ferredoxin, whether the hydrogenase or a short protein tag abrogated in vivo hydrogenase activity entirely (data not shown), likely due to the proximity of the ferredoxin C-terminus to the iron-sulfur cluster [41].

The behavior of the fusion protein is similar with the ferredoxin from $C$. acetobutylicum, although the bacterial ferredoxin is equally active when fused at either terminus (figure 5C). When fused to the hydrogenase C-terminus, the linker-length dependent activity displays similar characteristics to the spinach ferredoxin. Interestingly, when fused to the hydrogenase $\mathrm{N}$-terminus, in vivo activity with shorter linker lengths is improved. The putative ferredoxin-binding region is on the $\mathrm{N}$-terminal domain of the hydrogenase (figure 5A), which includes all of the F-clusters that transfer electrons from the surface to the active-site $\mathrm{H}$-cluster. Fusion with a short linker at the $\mathrm{C}$-terminus makes it impossible to reach the $\mathrm{N}$-terminal domain, resulting in the decreased activity compared to unfused proteins. At the N-terminus, however, a short linker still allows for interaction between the hydrogenase and its fused ferredoxin, resulting in increased activity.

\section{The effect of scaffolding ferredoxin and hydrogenase}

In signal transduction, complex protein scaffolds isolate pathways by localizing pathway components into a complex, directing the flow of information. These scaffolds can be rewired in their natural contexts in eukaryotic cells in order to alter the output behavior of signaling cascades $[43,44]$. Synthetic, modular scaffold proteins have been implemented in E. coli in order to direct flux in synthetic metabolic pathways, improving pathway output by up to 77 -fold [26]. These synthetic scaffolds are built from modular scaffold domains from eukaryotic signal transduction-PDZ, SH3, and GBD domainswhich tightly bind cognate ligand peptides that can be fused to any protein of interest for localization to the scaffold. We imported these scaffold designs into our synthetic pathway and found that scaffolding of the hydrogenase and ferredoxin dramatically affected the function of the pathway.

Scaffolding of metabolic pathways with small-molecule intermediates can lead to a "pipeline" effect, where the increase in the local concentration of upstream intermediates can speed up the reaction. This can significantly affect the pathway output, particularly when the chemical intermediates of the reaction pathway are toxic to the cell and increased throughput can lead to cell death if the intermediate is not rapidly converted by the next enzymatic step in the pathway [26]. Instead of small molecule intermediates, our synthetic pathway relies on proteinprotein interactions, as is the case in many signal transduction cascades. By channeling electron transfer through scaffolded interactions, the flux through the synthetic circuit can potentially be significantly increased in an insulated manner. Because the tertiary structures of the synthetic scaffolds have not been determined, however, it is also possible that the requirement of proteinprotein interaction for pathway function may lead to a decrease in pathway flux due to non-optimal insulation of interacting partners. We sought to characterize the effects of different scaffold structures, ligands, and linker lengths on the function of the synthetic PFOR-ferredoxin-hydrogenase circuit with ferredoxin and hydrogenase localized to the synthetic scaffold. All experiments were performed using the PFOR from $D$. africanus, and ferredoxin and hydrogenase from C. acetobutylicum.

The scaffold is an artificial protein built up of several modular peptide binding domains. The GBD binding domain is at the N-terminus, followed by the SH3 domain and the PDZ domain at the $\mathrm{C}$-terminus. The number of domains in each case is variable, and in Dueber et. al., the ratio of the domains to one another made significant differences in flux depending on the stoichiometry of the reactions in the synthetic pathway [26]. Although multiple diffusion-limited metabolic pathways could be enhanced using this design [26], artificially scaffolded redox pathways have not yet been investigated. While we were primarily interested in exploring how different configurations of scaffold domains and linker lengths affected the interaction of the redox 


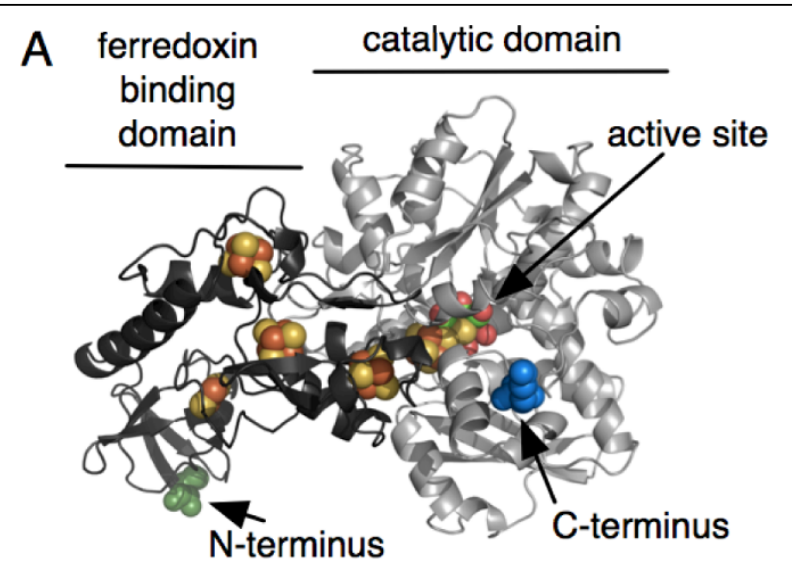

B

\author{
linker length \\ (\# of amino acids)
}

2142446104

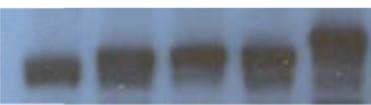

C

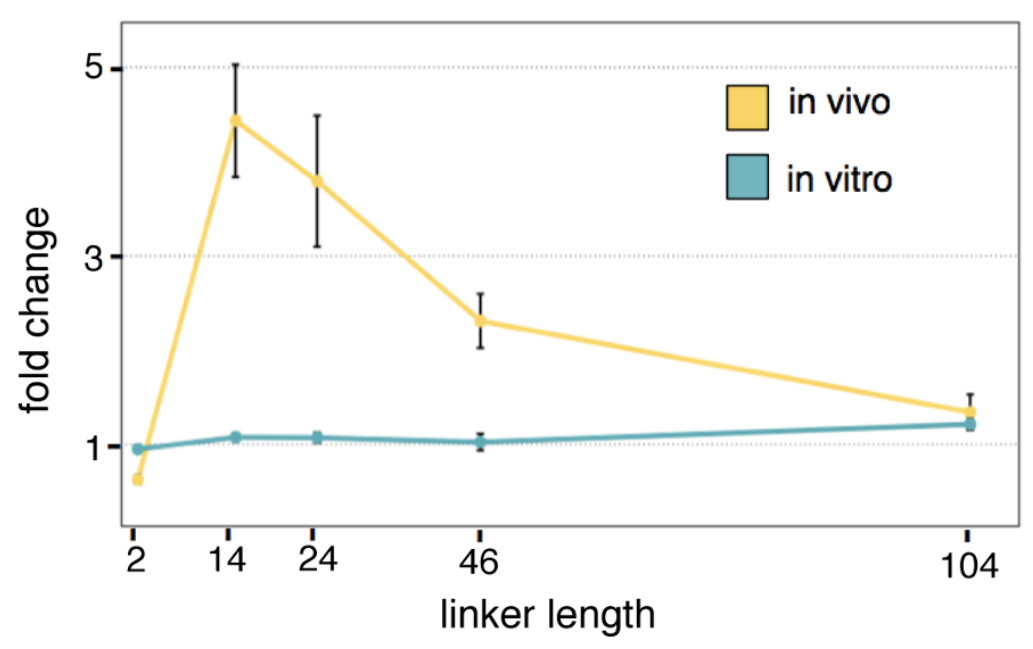

D

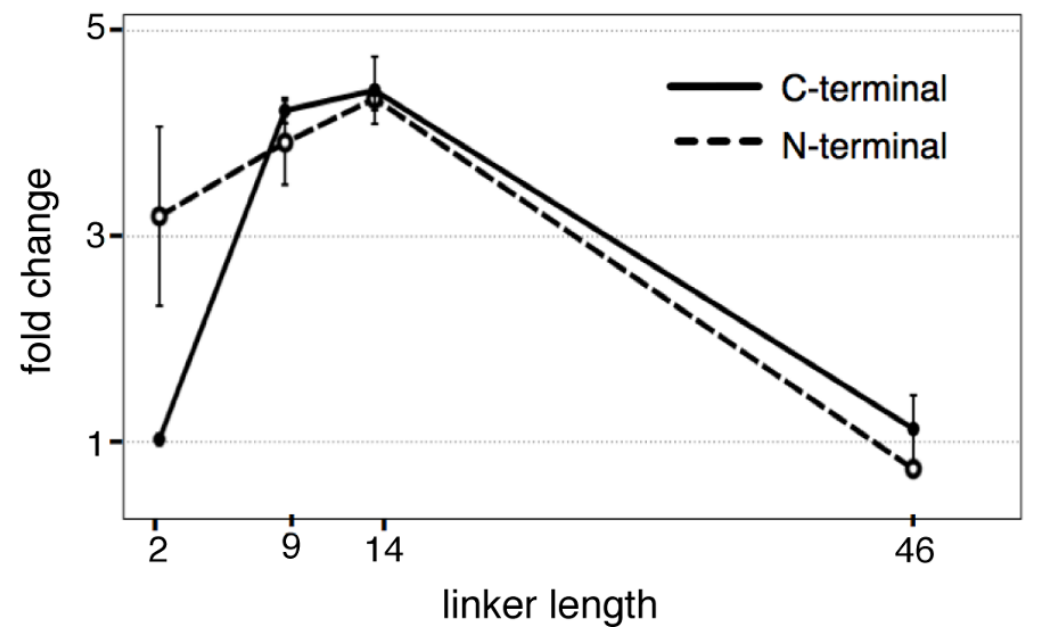

Figure 5 Increase in hydrogen production by hydrogenase-ferredoxin fusion A.) Schematic model of the protein fusion, here showing the C. acetobutylicum hydrogenase fused to the spinach ferredoxin $\mathrm{N}$-terminus ( $\mathrm{N}$-termini highlighted in blue, C-termini in green, and iron-sulfur clusters in orange). B.) Hydrogenase-ferredoxin fusion proteins are highly expressed and are the predicted size for the chimera, as indicated by western blotting with an anti-ferredoxin antibody. C.) Linker-length dependent behavior of fusion with spinach ferredoxin to hydrogenase C-terminus in vivo or in vitro. D.) Linker-length dependent behavior of fusion with Clostridium ferredoxin at the hydrogenase N-or C-terminus in vivo. 
proteins and therefore hydrogen output, we measured a three-fold improvement of hydrogen production in the scaffolded vs. non-scaffolded conditions when all of the proteins were expressed off of the lower activity pTet promoter, leading to a decrease in the absolute value of hydrogen production when compared to the Duet vectors (data not shown).

The ratio of the different scaffold domains, the ligand bound to the pathway components, and the length of the linker between the ligand and the ferredoxin protein all had significant effects on the output of the synthetic circuit (figure 6). Because ferredoxin and hydrogenase need to physically interact for the circuit to function, suboptimal configurations for the protein scaffold could conceivably sequester these proteins from one another. Indeed, we found that hydrogen output was decreased when the hydrogenase and ferredoxin were bound farther from one another along the length of scaffold (figure 6A).
Furthermore, the length of the linker connecting ferredoxin with the SH3 ligand also significantly affected the ability of the hydrogenase and ferredoxin to interact while bound to the scaffold. Increasing the linker length from five amino acids to twenty led to a 3-5 fold increase in hydrogen output from the scaffolded circuit (figure 6B). Linkers of intermediate length produced intermediate pathway output.

The ratio of GBD, SH3, and PDZ domains that made up the synthetic scaffold also significantly affect the function of the pathway in some cases. The stoichiometry of the hydrogen production reaction requires two ferredoxins for the reduction of a single hydrogen molecule, so it might be expected that scaffolds that localize more ferredoxin molecules will increase flux through the hydrogenase. Unfortunately, however, PFOR is substrate limited [45], with increasing concentrations of ferredoxin leading to decreased enzymatic activity. Increasing the ratio of
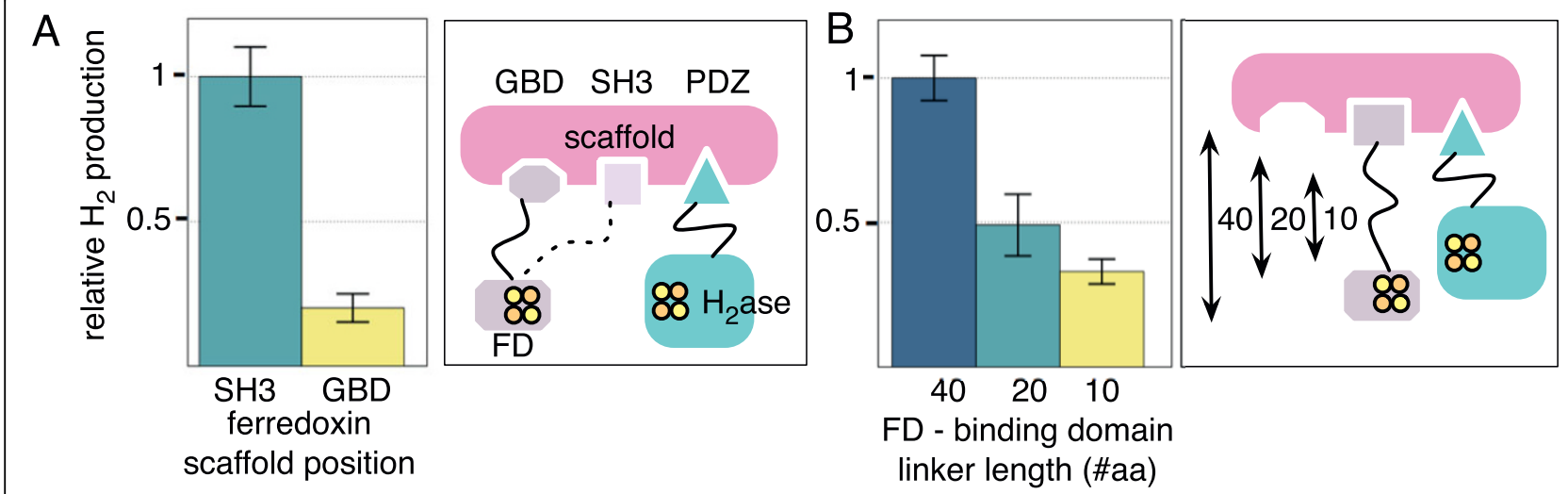

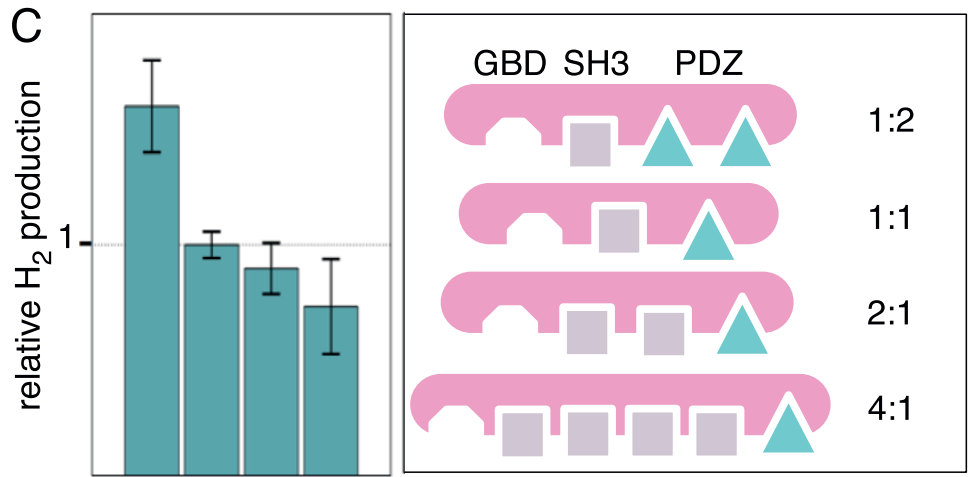

$1: 2$ 1:1 $2: 14: 1$

FD: $\mathrm{H}_{2}$ ase ratio

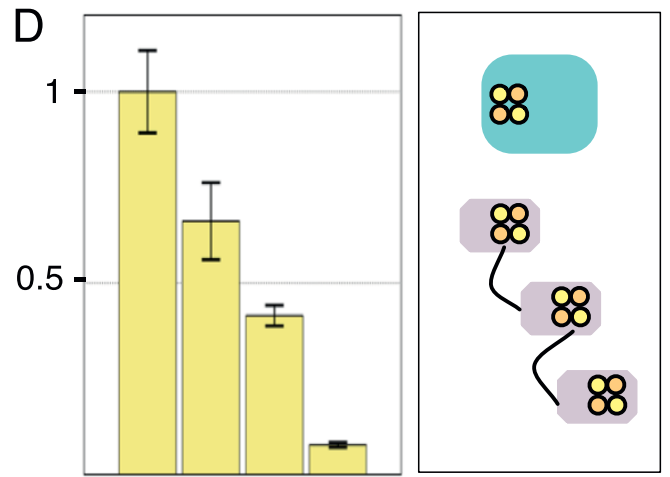

$1: 1 \quad 2: 1 \quad 3: 1 \quad 4: 1$

FD: $\mathrm{H}_{2}$ ase ratio

Figure 6 Effect of artificial scaffolding configuration on hydrogen production from the synthetic circuit. A.) Positional effects of ferredoxin targeting to artificial scaffold on hydrogen production. B.) Circuit efficiency is dependent upon length of flexible linker connecting ferredoxin (FD) to scaffolding. C.) Modulation of ferredoxin to hydrogenase ratio on scaffold affects hydrogen production, with decreasing yield observed at higher ferredoxin:hydrogenase ratios. D.) Direct fusion of ferredoxins to one another yields diminishing hydrogen production with increased numbers of fused ferredoxin proteins. 
ferredoxin:hydrogenase bound to the scaffold decreases the output from the synthetic pathway (figure $6 \mathrm{C}$ ). This effect was also seen when ferredoxin genes were fused in tandem without a scaffold, with output from the hydrogen production circuit steadily dropping with each added ferredoxin (figure 6D). This strategy may, however, increase pathway flux in other synthetic electron transfer pathways when the electron donor is not substrate limited.

\section{Discussion}

Metabolic engineering to produce biofuels must necessarily involve the redirection of reducing equivalents into the fuel molecule and away from other cellular metabolites. In cells, reducing equivalents are primarily stored in iron-sulfur cluster proteins and in small molecules such as NADPH, NADH, and $\mathrm{FADH}_{2}$. While the small molecules can freely diffuse through the cell and interact with a wide variety of enzymes, iron-sulfur proteins can be isolated through the techniques of metabolic and protein engineering. In the experiments described here, we applied four approaches to controlling electron flow out of the iron-sulfur cluster protein ferredoxin: deletion of potential interaction partners, enhancement of interaction by engineering of a protein surface, and increasing the local concentration of interacting proteins using a flexible peptide linker or attachment to a scaffold protein.

To measure the effect of these approaches, we first created an artificial pathway to produce the biofuel hydrogen in E. coli. The pathway consists of the proteins pyruvate-ferredoxin oxidoreductase (PFOR), ferredoxin, and a hydrogenase (expressed in the presence of hydrogenase maturation factors). This pathway produces a theoretical maximum of two molecules of hydrogen per input glucose, and still allows acetyl-CoA production from pyruvate. We characterized the relative efficacy of hydrogen production using various combinations of PFOR, ferredoxin, and hydrogenase molecules from different species, and found that PFOR from $D$. africanus in combination with ferredoxin and hydrogenase from C. acetobutylicum was the most active pathway, predicted in part by previous in vitro data $[18,34,46]$.

To direct electron flow from ferredoxin into hydrogenase, we first deleted genes encoding six other proteins with which PFOR and/or ferredoxin might interact. Of these, only deletion of $y d b K$, encoding a putative $E$. coli PFOR, resulted in enhanced hydrogen production. In addition, in the absence of the PFOR from $D$. africanus, deletion of $y d b K$ resulted in a decrease in the background level of hydrogen. These results provide further evidence that $y d b K$ is a functional PFOR that can interact with a variety of electron acceptors, particularly the spinach ferredoxin [20].

As a converse approach, we addressed whether the levels of hydrogen production could be enhanced by improving the binding between ferredoxin and hydrogenase in vivo. In this case we used the $C$. reinhardtii hydrogenase, whose interaction with plant-type ferredoxin has been computationally modelled by Long et al., who suggested mutations that could enhance this interaction [25]. Several mutations that improve charge complementarity between spinach ferredoxin and this hydrogenase were found to enhance electron transfer between these proteins in vivo, as inferred by increased hydrogen production. These results indicate that the activity of the hydrogenase is limited, in part, by its ability to interact with ferredoxin; i.e. that the collision and docking of these proteins is not effectively in excess.

Finally, we used two different methods to increase the relative local concentration of ferredoxin and hydrogenase: direct fusion by a flexible glycine/serine-rich linker, and indirect fusion by attachment of these proteins to interaction modules that bind to a common scaffold. Each approach significantly improved hydrogen production in a strongly configuration-dependent manner in $v i v o$, as expected if the ferredoxin and hydrogenase were primarily interacting in cis. For example, when the ferredoxin and hydrogenase were attached by a linker too short to allow in cis interaction, hydrogen production was relatively low, but increased significantly when the linker was long enough to allow interaction. As the attaching linker was further lengthened, hydrogen production decreased gradually, consistent with the two proteins occupying a sphere of increasing volume and decreasing relative concentration. As a tactic for metabolic engineering, protein fusion and/or scaffolding is particularly useful with iron-sulfur cluster proteins, because their electrons must be transferred protein-toprotein-no small molecule carriers of reducing equivalents are generated that might diffuse away.

The iron-sulfur proteins in our synthetic circuit present a modular system, with proteins from disparate species able to interact and produce high levels of hydrogen. Such modular systems are valuable for further synthetic biological manipulation and experimentation. The synthetic pathway presented here is a relatively simple method for the analysis of activities and electron transfer properties of hydrogenases, ferredoxins, and PFOR genes from any number of species, or engineered synthetic electron transfer proteins. These in vivo data are a valuable complement to in vitro binding constants and kinetic parameters of the enzymes and will be useful in further designing and optimizing microbial systems for hydrogen production.

Such synthetic biological systems can also be used to better understand biological electron transfer systems. The role of ferredoxins in E. coli metabolism is poorly characterized, with ferredoxins performing many unknown but required functions in the cell. Here we 
tested deletions of six iron-sulfur proteins expected to interact with ferredoxins, many of which are previously uncharacterized. While only one gene deletion $(\Delta y d b K)$ affected our specific hydrogen production pathway, combinatorial deletions may affect hydrogen production in different ways, or may affect other synthetic electron transfer pathways. Further deletions of iron-sulfur oxidoreductases and combinations thereof may lead to a more complete understanding of electron transfer systems in the E. coli cytoplasm, as well as the development of a host strain for expression of heterologous electron transfer pathways for synthetic biology. Such a strain would have to retain the ability to mature ironsulfur clusters but limit the function of proteins that can interact with ferredoxins and ferredoxin oxidoreductases to ensure optimal electron flux through the synthetic pathway. Such specialized strains of $E$. coli may be optimized for other types of synthetic pathway designs and may be better equipped for industrial purposes than proposed "minimal" cells [47], as they would retain many of the mechanisms that allow for robust growth and protein expression.

Our pathway can also be used to further analyze protein-protein interaction surfaces for electron transfer, including for mutagenic studies to determine the binding surface on the clostridial hydrogenases, which is poorly understood. An improved understanding of the electron transfer surface between the hydrogenase and ferredoxin would significantly affect our picture of how electron transfer pathways co-evolved; whether specific ferredoxins evolved for interaction with specific enzymes or whether electron transfer is regulated in other ways. Considering the ability of ferredoxins from many distant species to interact with various hydrogenases, the impact of further binding surface optimization may be negligible, or may require co-evolution of complementary mutations on both binding partners to result in highly specific interactions.

Iron-sulfur proteins are also uniquely suited to isolation techniques that involve physical scaffolding. Electrons tunnel between proteins in close proximity, so direct protein fusion improves the local concentration of electron transfer proteins and thus improves the electron transfer rates. This is in contrast to other synthetic metabolic pathways with small molecule intermediates, whose diffusion through the cellular environment is much faster, limiting the potential improvement by protein fusion. This method can be easily adapted to other electron transfer pathways in a modular, extensible manner. Moreover, the dependence of hydrogenase activity upon scaffold design and/or the links between the ligands and scaffold illustrates that synthetic redox pathways can be coupled through interaction with a common adaptor protein in order to modulate electron flux through the system. Unlike reactions with diffusible intermediates, scaffolding of redox partners requires that the scaffold design allow sterically unhindered interaction between bound protein to enable electrons to tunnel between closely apposed iron-sulfur clusters. Incorrect design may tether redox partners in a manner that constrains them and prevents electron transfer, as we observed when hydrogenase and ferredoxin were bound to domains on distal ends of the scaffold. Through attention to scaffold design, further optimization may significantly improve hydrogen production through the synthetic circuit, as well as provide a template for future scaffolding of other electron transfer pathways.

Biological hydrogen production is a promising and well-studied system for sustainable energy production. The insulation approaches presented here are widely applicable to other biological hydrogen systems, from in vitro enzymatic pathways [11], where protein fusions would likely improve kinetic rates, to methods for boosting natural hydrogen production in heterotrophic [48] and photosynthetic $[49,50]$ organisms. Photosynthetic systems in particular may benefit from the insulation strategies presented here, as ferredoxins are the primary electron carrier in photosynthetic organisms and competition for electrons from other metabolic pathways is strong. Improvement of protein binding in plant-type ferredoxins may be useful in such systems, as well as the applicability of protein fusion or pathway scaffolding to a wide range of biological systems.

The three methods described here - deletion of competing reactions, optimization of interaction surfaces, and protein tethering - could be combined with each other and with other strategies for optimizing redox pathways. Direct fusion of proteins with a flexible linker had the largest effect by itself, and simultaneous deletion of competing reactions should have a multiplicative effect since these involve independent aspects of the pathway. Combining protein fusion with optimization of protein interaction surfaces may have a sub-multiplicative effect because both approaches affect the same pathway step.

Alone, the pathway presented here has a maximum theoretical yield of $16.67 \%$, with two hydrogen molecules produced for every molecule of glucose. Our in vivo hydrogen production system produced hydrogen at levels that ranged from $0.005 \%$ to nearly $3 \%$ of this theoretical maximum. Combination of methods presented here with other metabolic engineering approaches will likely increase hydrogen production from pyruvate closer to the theoretical yield. In particular, a background strain with deletions in several genes that either take electrons away from the pathway, limit iron sulfur cluster biogenesis, or use glycolytic flux from pyruvate 
would drastically increase hydrogen production. Such an engineered strain would be have deletions in $y d b K$, shown here to boost hydrogen production, as well as in $i s c R$, a negative regulator of iron sulfur cluster biogenesis shown to limit hydrogen production from heterologously expressed [FeFe]-hydrogenases [35]. Flux through PFOR could be increased through the deletions of pyruvate metabolism genes such as the pyruvate dehydrogenase complex and pyruvate formate lyase, as well as other metabolic enzymes that consume pyruvate, which when deleted have led to accumulation of large quantities of pyruvate in $E$. coli [51]. Addition of optimized hydrogen production through natural $[\mathrm{NiFe}]$-hydrogenase pathways, deletion of uptake hydrogenase genes [52], and incorporation of other ferredoxin-oxidoreductases that generate electrons from other metabolic conversions [19] is likely to bring hydrogen production closer to the absolute theoretical maximum of 12 moles of hydrogen per mole of glucose.

\section{Conclusions}

Electron transfer systems such as our hydrogenase pathway are an untapped resource for synthetic biology, which seeks to design biological pathways as predictably as electronic circuits [53]. Electrons are unique metabolites whose movement in biological systems occurs by quantum-mechanical tunneling between protein-bound cofactors such as iron-sulfur clusters. As a result, escape by diffusion into an aqueous phase is avoided, offering distinctive opportunities for control. The circuit described here moves electrons from higher to lower energy, while performing work in the form of hydrogen production. The rationally constructed insulation of the pathway through elimination of side reactions, interaction surface optimization, and protein fusion or scaffolding indicate that all four methods are viable for synthetic circuit design and all strategies may play a role in the evolution of complex isolated circuits in natural metabolism. This type of synthetic-biological analysis may yield insights into natural mechanisms for controlling electron flow, and may provide new approaches for metabolic engineering and bioenergy.

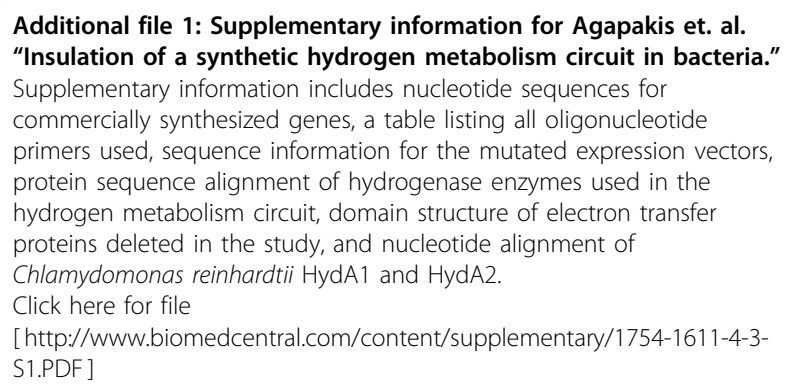

\section{Acknowledgements}

We would like to acknowledge David Savage, Devin Burrill, Bruno Afonso, Noah Taylor, and Dominique Frueh for helpful discussion and comments on the manuscript, Craig Blain, Renan Antonio Escalante Chung, and Bennett Caughey for technical assistance, and John Dueber, Matthew Posewitz, Colleen Hansel, Laetitia Pieulle, and Kenneth Noll for providing materials. This material is based upon work supported by the Army Research Office Award No. W911NF-09-1-0226. CMA and EHW are supported by NSF Graduate Research fellowships and PMB is funded by the Harvard University Center for the Environment Graduate Consortium.

\section{Author details}

'Department of Systems Biology, Harvard Medical School, Boston, MA 02115 USA. ${ }^{2}$ Wyss Institute for Biologically Inspired Engineering, Harvard University, Boston, MA 02115, USA.

\section{Authors' contributions}

CMA, DCD, PMB, EHW, JCW, and PAS designed experiments and analyzed data; CMA, DCD, PMB, EHW performed experiments; CMA, DCD, JCW, and PAS wrote the paper. All authors read and approved the final manuscript.

\section{Competing interests}

The authors declare that they have no competing interests.

Received: 22 December 2009 Accepted: 25 February 2010

Published: 25 February 2010

\section{References}

1. Marcus RA, Sutin N: Electron transfers in chemistry and biology. Biochim Biophys Acta 1985, 811:265-322.

2. Page CC, Moser CC, Chen X, Dutton PL: Natural engineering principles of electron tunnelling in biological oxidation-reduction. Nature 1999, 402:47-52.

3. Varadarajan R, Zewert TE, Gray HB, Boxer SG: Effects of buried ionizable amino acids on the reduction potential of recombinant myoglobin. Science 1989, 243:69-72.

4. Lim W: The modular logic of signaling proteins: building allosteric switches from simple binding domains. Curr Opin Struct Biol 2002, 12:61-68.

5. Gou P, Hanke GT, Kimata-Ariga Y, Standley DM, Kubo A, Taniguchi I, Nakamura $\mathrm{H}$, Hase T: Higher order structure contributes to specific differences in redox potential and electron transfer efficiency of root and leaf ferredoxins. Biochemistry 2006, 45:14389-14396.

6. Gilardi G, Fantuzzi A, Sadeghi SJ: Engineering and design in the bioelectrochemistry of metalloproteins. Curr Opin Struct Biol 2001, 11:491-499.

7. Wong TS, Schwaneberg U: Protein engineering in bioelectrocatalysis. Current Opinion in Biotechnology 2003, 14:590-596.

8. Sadeghi SJ, Meharenna YT, Fantuzzi A, Valetti F, Gilardi G: Engineering artificial redox chains by molecular 'Lego'. Faraday Disc 2000, 116:135-153.

9. Bhalerao KD: Synthetic gene networks: the next wave in biotechnology? Trends Biotechnol 2009, 27:368-374.

10. Ghirardi ML, Posewitz MC, Maness PC, Dubini A, Yu J, Seibert M: Hydrogenases and Hydrogen Photoproduction in Oxygenic Photosynthetic Organisms. Annu Rev Plant Biol 2007, 58:71-91.

11. Ye X, Wang Y, Hopkins RC, Adams MWW, Evans BR, Mielenz JR, Zhang Y-HP: Spontaneous High-Yield Production of Hydrogen from Cellulosic Materials and Water Catalyzed by Enzyme Cocktails. ChemSusChem 2009, 2:149-152.

12. Aguirre de Carcer I, DiPasquale A, Rheingold AL, Heinekey DM: Active-site models for iron hydrogenases: reduction chemistry of dinuclear iron complexes. Inorganic chemistry 2006, 45:8000-8002.

13. Yoshida A, Nishimura T, Kawaguchi H, Inui M, Yukawa H: Efficient induction of formate hydrogen lyase of aerobically grown Escherichia coli in a three-step biohydrogen production process. Appl Microbiol Biotechnol 2007, 74:754-760. 
14. Maeda T, Sanchez-Torres V, Wood TK: Protein engineering of hydrogenase 3 to enhance hydrogen production. Appl Microbiol Biotechnol 2008, 79:77-86.

15. Akhtar $M$, Jones $P$ : Engineering of a synthetic hydF-hydE-hydG-hydA operon for biohydrogen production. Anal Biochem 2008, 373:170-172.

16. Laurinavichene TV, Tsygankov AA: H2 consumption by Escherichia coli coupled via hydrogenase 1 or hydrogenase 2 to different terminal electron acceptors. FEMS Microbiol Lett 2001, 202:121-124.

17. Sawers RG: Formate and its role in hydrogen production in Escherichia coli. Biochem Soc Trans 2005, 33:42-46.

18. King PW, Posewitz MC, Ghirardi ML, Seibert M: Functional studies of [FeFe] hydrogenase maturation in an Escherichia coli biosynthetic system. $J$ Bacteriol 2006, 188:2163-2172.

19. Veit A, Akhtar M, Mizutani T, Jones PR: Constructing and testing the thermodynamic limits of synthetic NAD(P)H:H 2pathways. Microbial Biotechnology 2008, 1:382-394.

20. Akhtar M, Jones PR: Construction of a synthetic YdbK-dependent pyruvate:H2 pathway in Escherichia coli BL21(DE3). Metab Eng 2009, 11:139-147.

21. Richard DJ, Sawers G, Sargent F, McWalter L, Boxer DH: Transcriptional regulation in response to oxygen and nitrate of the operons encoding the [NiFe] hydrogenases 1 and 2 of Escherichia coli. Microbiology 1999, 145:2903-2912.

22. Skerker J, Perchuk B, Siryaporn A, Lubin E, Ashenberg O, Goulian M, Laub M: Rewiring the Specificity of Two-Component Signal Transduction Systems. Cell 2008, 133:1043-1054.

23. Akashi T, Matsumura T, Ideguchi T, Iwakiri K, Kawakatsu T, Taniguchi I, Hase T: Comparison of the electrostatic binding sites on the surface of ferredoxin for two ferredoxin-dependent enzymes, ferredoxin-NADP(+) reductase and sulfite reductase. J Biol Chem 1999, 274:29399-29405.

24. Peters JW, Lanzilotta WN, Lemon BJ, Seefeldt LC: X-ray crystal structure of the Fe-only hydrogenase $(\mathrm{Cpl})$ from Clostridium pasteurianum to 1.8 angstrom resolution. Science 1998, 282:1853-1858.

25. Long H, King P, Ghirardi M, Kim K: Hydrogenase/Ferredoxin ChargeTransfer Complexes: Effect of Hydrogenase Mutations on the Complex Association. The journal of physical chemistry 2009, 113:4060-4067.

26. Dueber JE, Wu GC, Malmirchegini GR, Moon TS, Petzold CJ, Ullal AV, Prather KL, Keasling JD: Synthetic protein scaffolds provide modular control over metabolic flux. Nat Biotechnol 2009, 27:753-759.

27. Guerrini O, Burlat B, Léger C, Guigliarelli B, Soucaille P, Girbal L: Characterization of Two 2[4Fe4S] Ferredoxins from Clostridium acetobutylicum. Curr Microbiol 2008, 56:261-267.

28. Pieulle L, Magro V, Hatchikian EC: Isolation and analysis of the gene encoding the pyruvate-ferredoxin oxidoreductase of Desulfovibrio africanus, production of the recombinant enzyme in Escherichia coli, and effect of carboxy-terminal deletions on its stability. Journal of Bacteriology 1997, 179:5684-5692.

29. Phillips I, Silver P: A New Biobrick Assembly Strategy Designed for Facile Protein Engineering. dspace 2006.

30. Cironi P, Swinburne I, Silver P: Enhancement of Cell Type Specificity by Quantitative Modulation of a Chimeric Ligand. Journal of Biological Chemistry 2008, 283:8469-8476.

31. Baba T, Ara T, Hasegawa M, Takai Y, Okumura Y, Baba M, Datsenko KA, Tomita M, Wanner BL, Mori H: Construction of Escherichia coli K-12 inframe, single-gene knockout mutants: the Keio collection. Mol Syst Biol 2006, 2:0008.

32. Guex N, Peitsch MC: SWISS-MODEL and the Swiss-PdbViewer: an environment for comparative protein modeling. Electrophoresis 1997, 18:2714-2723.

33. Boxma B, de Graaf RM, Staay van der GW, van Alen TA, Ricard G, Gabaldón T, van Hoek AH, Moon-van der Staay SY, Koopman WJ, van Hellemond JJ, et al: An anaerobic mitochondrion that produces hydrogen. Nature 2005, 434:74-79.

34. Moulis JM, Davasse V: Probing the role of electrostatic forces in the interaction of Clostridium pasteurianum ferredoxin with its redox partners. Biochemistry 1995, 34:16781-16788.

35. Akhtar MK, Jones PR: Deletion of iscR stimulates recombinant clostridial Fe-Fe hydrogenase activity and $\mathrm{H} 2$-accumulation in Escherichia coli BL21(DE3). Appl Microbiol Biotechnol 2008, 78:853-862.

36. Yonekura-Sakakibara K, Onda Y, Ashikari T, Tanaka Y, Kusumi T, Hase T: Analysis of reductant supply systems for ferredoxin-dependent sulfite reductase in photosynthetic and nonphotosynthetic organs of maize. Plant Physiol 2000, 122:887-894.

37. McCord JM: The evolution of free radicals and oxidative stress. Am J Med 2000, 108:652-659

38. Zarrinpar A, Park SH, Lim WA: Optimization of specificity in a cellular protein interaction network by negative selection. Nature 2003, 426:676-680.

39. Chang $\mathrm{CH}$, King PW, Ghirardi ML, Kim K: Atomic resolution modeling of the ferredoxin:[FeFe] hydrogenase complex from Chlamydomonas reinhardtii. Biophys J 2007, 93:3034-3045.

40. Long H, Chang CH, King PW, Ghirardi ML, Kim K: Brownian Dynamics and Molecular Dynamics Study of the Association between Hydrogenase and Ferredoxin from Chlamydomonas reinhardtii. Biophysical Journal 2008, 95:3753-3766.

41. Aliverti A, Zanetti G: A three-domain iron-sulfur flavoprotein obtained through gene fusion of ferredoxin and ferredoxin-NADP+ reductase from spinach leaves. Biochemistry 1997, 36:14771-14777.

42. Krishnamurthy VM, Semetey V, Bracher PJ, Shen N, Whitesides GM: Dependence of effective molarity on linker length for an intramolecular protein-ligand system. J Am Chem Soc 2007, 129:1312-1320.

43. Bashor CJ, Helman NC, Yan S, Lim WA: Using Engineered Scaffold Interactions to Reshape MAP Kinase Pathway Signaling Dynamics. Science 2008, 319:1539-1543.

44. Park SH, Zarrinpar A, Lim WA: Rewiring MAP kinase pathways using alternative scaffold assembly mechanisms. Science 2003, 299:1061-1064.

45. Uyeda K, Rabinowitz JC: Pyruvate-ferredoxin oxidoreductase. IV. Studies on the reaction mechanism. J Biol Chem 1971, 246:3120-3125.

46. Pieulle L, Charon MH, Bianco P, Bonicel J, Pétillot Y, Hatchikian EC: Structural and kinetic studies of the pyruvate-ferredoxin oxidoreductase/ ferredoxin complex from Desulfovibrio africanus. Eur J Biochem 1999, 264:500-508.

47. Forster AC, Church GM: Towards synthesis of a minimal cell. Mol Syst Biol 2006, 2:45.

48. Maeda, Sanchez-Torres, Wood: Enhanced hydrogen production from glucose by metabolically engineered Escherichia coli. Appl Microbiol Biotechnol 2007, 77:879-890.

49. Cournac L, Guedeney G, Peltier G, Vignais PM: Sustained photoevolution of molecular hydrogen in a mutant of Synechocystis sp. strain PCC 6803 deficient in the type I NADPH-dehydrogenase complex. J Bacteriol 2004, 186:1737-1746.

50. Melis A, Zhang L, Forestier M, Ghirardi ML, Seibert M: Sustained photobiological hydrogen gas production upon reversible inactivation of oxygen evolution in the green alga Chlamydomonas reinhardtii. Plant Physiol 2000, 122:127-136.

51. Zhu $Y$, Eiteman MA, Altman R, Altman E: High Glycolytic Flux Improves Pyruvate Production by a Metabolically Engineered Escherichia coli Strain. Appl Environ Microbiol 2008, 74:6649-6655.

52. Penfold DW, Sargent F, Macaskie LE: Inactivation of the Escherichia coli $\mathrm{K}-12$ twin-arginine translocation system promotes increased hydrogen production. FEMS Microbiol Lett 2006, 262:135-137.

53. Andrianantoandro E, Basu S, Karig D, Weiss R: Synthetic biology: new engineering rules for an emerging discipline. Mol Syst Biol 2006, 2, 2006.0028.

54. Binda C, Coda A, Aliverti A, Zanetti G, Mattevi A: Structure of the mutant E92K of [2Fe-2S] ferredoxin I from Spinacia oleracea at $1.7 \mathrm{~A}$ resolution. Acta Crystallogr D Biol Crystallogr 1998, 54:1353-1358.

doi:10.1186/1754-1611-4-3

Cite this article as: Agapakis et al:: Insulation of a synthetic hydrogen metabolism circuit in bacteria. Journal of Biological Engineering 2010 4:3 